\title{
AUTOMATED CLASSIFICATION OF LAND COVER USING LANDSAT 8 OLI SURFACE REFLECTANCE PRODUCT AND SPECTRAL PATTERN ANALYSIS CONCEPT - CASE STUDY IN HANOI, VIETNAM
}

\author{
Duong Nguyen Dinh \\ Institute of Geography, Vietnam Academy of Science and Technology, 18 Hoang Quoc Viet Rd., Cau Giay, Hanoi, Vietnam - \\ duong.nguyen2007@gmail.com \\ Commission VIII, WG VIII/8
}

KEY WORDS: Land cover, Landsat 8 surface reflectance, pattern analysis, automated classification

\begin{abstract}
:
Recently USGS released provisional Landsat 8 Surface Reflectance product, which allows conducting land cover mapping over large composed of number of image scenes without necessity of atmospheric correction. In this study, the authors present a new concept for automated classification of land cover. This concept is based on spectral patterns analysis of reflected bands and can be automated using predefined classification rule set constituted of spectral pattern shape, total reflected radiance index (TRRI) and ratios of spectral bands.

Given a pixel vector $B_{6}=\left\{b_{1}, b_{2}, b_{3}, b_{4}, b_{5}, b_{6}\right\}$ where $b_{1}, b_{2}, \ldots, b_{6}$ denote bands $2,3, \ldots, 7$ of OLI sensor respectively. By using the pixel vector $B_{6}$ we can construct spectral reflectance curve. Each spectral curve is featured by a shape, which can be described in simplified form of an analogue pattern, which is consisted of 15 digits of 0,1 and 2 showing mutual relative position of spectral vertices. Value of comparison between band $i$ and $j$ is 2 if $b_{j}>b_{i}, 1$ if $b_{j}=b_{i}$ and 0 if $b_{j}<b_{i}$. Simplified spectral pattern is defined by 15 digits as $m_{1,2} m_{1,3} m_{1,4} m_{1,5} m_{1,6} m_{2,3} m_{2,4} m_{2,5} m_{2,6} m_{3,4} m_{3,5} m_{3,6} m_{4,5} m_{4,6} m_{5,6}$ where $m_{i, j}$ is result of comparison of reflectance between $b_{i}$ and $b_{j}$ and has values of 0,1 and 2 . After construction of SSP for each pixel in the input image, the original image will be decomposed to component images, which contain pixels with the same SRCS pattern. The decomposition can be written analytically by equation $A=\sum_{k=1}^{n} C_{k}$ where $\boldsymbol{A}$ stands for original image with 6 spectral bands, $\boldsymbol{n}$ is number of component images decomposed from $\boldsymbol{A}$ and $\boldsymbol{C}_{\boldsymbol{k}}$ is component image. For this study, we use Landsat 8 OLI reflectance image LC81270452013352LGN00 and LC81270452015182LGN00. For the decomposition, we use only six reflective bands. Each land cover class is defined by SSP code, threshold values for TRRI and band ratios. Automated classification of land cover was realized with 8 classes: forest, shrub, grass, water, wetland, develop land, barren and others. This paper provides a preliminary research result on application of multispectral image decomposition using simplified spectral pattern for classification of land cover for Hanoi area.
\end{abstract}

\section{INTRODUCTION}

Land use and land cover mapping is very important in global and regional environment study. Land use and land cover are pivotal data in various study such as earth-atmosphere interactions and biodiversity loss, sustainable development and human responses to global change and are important for integrated modelling and assessment of environmental issues in general (Turner et al., 1995). Land cover mapping by remotely sensed data is commonly carried out by either supervised or unsupervised classification. Both approaches require large amount of knowledge and skills of analysts about land cover conditions and the utilization of the specific software.

Recently requirement of automation of classification of land cover is getting gradually important, especially when NASA and ESA started to open all archived image data freely to public without any cost.

A novel and innovative automated processing environment for classification of land use and land cover by framework TWOPAC ( TWinned Object and Pixel based Automated classification Chain) was successfully implemented and used with several remotely sensed data as MODIS, SPOT, Landsat and Aster (Huth et al., 2012). There is a report on automated land cover mapping and independent change detection with Landsat TM and ETM+ data (Verhegghen etal., 2010). The study proposed method on combination of unsupervised classification and segmentation to support forest mapping in Africa. Another study on use of LIDAR together with high spatial resolution multispectral image data for automatic classification of land cover has been reported (Syed et al., 2005).

In this paper, the author presents novel approach on fully automated classification of land cover using the surface reflectance product of Landsat 8 OLI image data. The proposed concept is based on application of simplified spectral patterns SSP and implemented with Landsat 8 OLI data observed in 2013 and 2015 for Hanoi, Vietnam area.

\section{MATERIAL AND METHODS}

\subsection{Material}

In this study, surface reflectance product of Landsat 8 OLI scenes LC81270452013352LGN00 and LC81270452015182LGN00 was used. The surface reflectance product is at this time a provisional and is provided by USGS through website EarthExplorer by ordering. The Landsat 8 surface product was processed by the NASA GFC internal algorithm and at this moment, it is only provisional product. The SR data is packed in GeoTIFF format, which common format for every image analysis system. 


\subsection{Method}

Only six reflective bands of OLI were used for classification. They are band 2, 3, 4, 5, 6, and 7. Reflectance values of every pixel formulates spectral pattern as showed in Figure 2. Different land cover objects have different spectral pattern due to spectral characteristics of the materials.

To use spectral pattern in classification, we transform the spectral pattern to simplified spectral pattern SSP, which is defined by a string of 15 digits showing relation among reflectance values in each spectral bands.
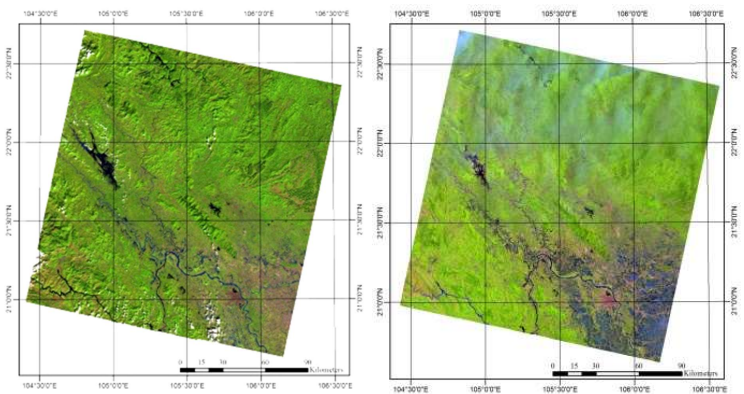

Figure 1. False colour composite bands 543: RGB of the scenes LC81270452013352LGN00 (left) and

LC81270452015182LGN00 (right). Hanoi city is located in right-bottom corner. The LC81270452013352LGN00 is almost cloud free while the LC81270452015182LGN00 is affected by thin cloud

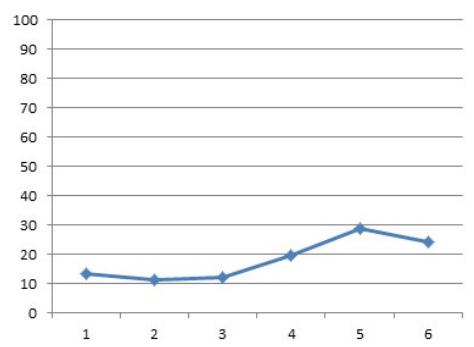

Figure 2. Spectral pattern of barren land in OLI image data Vertical axis shows reflectance values and horizontal axis shows OLI reflective band number

Given a pixel vector $B_{6}=\left\{b_{1}, b_{2}, b_{3}, b_{4}, b_{5}, b_{6}\right\}$ where $b_{1}$, $b_{2}, \ldots, b_{6}$ denote bands $2,3, \ldots, 7$ of OLI sensor respectively. Value of comparison between band $i$ and $j$ is 2 if $b_{j}>b_{i}, 1$ if $b_{j}=b_{i}$ and 0 if $b_{j}<b_{i}$. Simplified spectral pattern SSP is defined by 15 digits as

$m_{1,2} m_{1,3} m_{1,4} m_{1,5} m_{1,6} m_{2,3} m_{2,4} m_{2,5} m_{2,6} m_{3,4} m_{3,5} m_{3,6} m_{4,5} m_{4,6} m_{5,6}$

where $m_{i, j}$ is result of comparison of reflectance between $b_{i}$ and $b_{j}$ and has values of 0,1 and 2 .

Similar land cover objects have the same simplified spectral pattern. To classify a OLI surface reflectance product we need to develop at first a database of SSP and corresponding land cover type. To further separate objects which have the same SSP we use parameters in form of arithmetic combination of bands. By this way, each land cover object is digitally defined by a structure:
Class $\quad$ Full name of class

SSP Simplified spectral pattern

Thresholds of TRRI or ratios of spectral bands

Code Land cover code of class

Color $R, G, B$ to display the class

Name Short name

End Full name of class

This structure defines evergreen forest. T1 TRRI index (Duong 1998) computed for bands 2, 3 and 4. After classification, the pixel is labelled by code 42 and displayed by colour red $=0$, green $=176$ and blue $=80$.

In OLI scene, there are many SSP. Some SSP have high pixel frequency and the other low frequency. There are many SSP have frequency of one pixel only. The database is created for dominating SSP only. Therefore, after classification many pixels remain unknown. To classify the unknown pixel we use spectral matching technique. We compute spectral similarity values between the unknown pixel and known class which was defined in database. The unknown pixel will be assigned to class with smallest spectral similarity value SSV. SSV is computed by the following procedure (Homayouni 2004).

$$
S S V=\sqrt{E d^{2}+\left((1-\rho)^{2}\right)}
$$

Where Ed is normalized Euclidian distance between the unknown pixel and a land cover class. $\rho$ is the Pearson statistical correlation. They are computed by formulas:

$$
\begin{gathered}
E d_{\text {orig }}=\sqrt{\sum_{i=1}^{n}\left(t_{i}-p_{i}\right)^{2}} \\
E d=\frac{\left(E d_{\text {orig }}-m\right)}{(M-m)} \\
\rho=\frac{1}{n-1}\left[\frac{\sum_{i=1}^{n}\left(t_{i}-\mu_{i}\right)\left(p_{i}-\mu_{p}\right)}{\sigma_{i} \sigma_{p}}\right]
\end{gathered}
$$

The spectral matching is carried out for all unknown pixels.
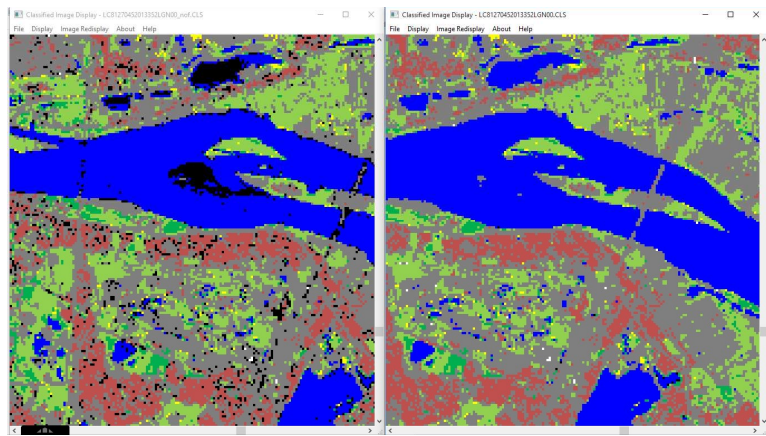

Figure 2. Classified image without filling of unknown pixels (left) and with filling of unknown pixels by spectral matching (right)

\section{RESULTS}

To implement automated classification of land cover, we developed software running in MSDOS command mode. The program requires two input files: metadata file of the OLI surface reflectance product and a table of land cover 
classification definition. The table of land cover definition contains 71 subclasses ranging from water to barren land. The final land cover result, however, has less number of classes such as water, wetland, forest, shrub land, grassland, barren land, cloud, and cloud shadow. Though there are 999 SSP in the selected scene, but the first 30 SSP occupies $96.3 \%$ of total number of pixels in the scene. The table of land cover definition was developed using only few SSP, which dominates pixel population of the given scene. Table 1 shows the most dominating SSP in the scene LC81270452013352LGN00.

\begin{tabular}{|c|c|c|}
\hline $\begin{array}{l}\text { Simplified } \\
\text { Spectral pattern } \\
\text { SSP }\end{array}$ & $\begin{array}{ll}\text { Number } & \text { of } \\
\text { pixels } & \text { of } \\
\text { SSP } & \end{array}$ & $\begin{array}{l}\text { Percentage } \\
{[\%]}\end{array}$ \\
\hline 222220222222000 & 22642414 & 55.8911 \\
\hline 222222222222000 & 4295952 & 10.6042 \\
\hline 202220222222000 & 3495831 & 8.6292 \\
\hline 212220222222000 & 1922604 & 4.7458 \\
\hline 222222222222200 & 1612062 & 3.9793 \\
\hline 222221222222000 & 609013 & 1.5033 \\
\hline 222220220222000 & 424727 & 1.0484 \\
\hline 222222220220000 & 391890 & 0.9674 \\
\hline 202220220222000 & 378192 & 0.9335 \\
\hline 222220220220000 & 377703 & 0.9323 \\
\hline
\end{tabular}

Table 1. Dominating SSP in the scene LC81270452013352LGN00

Pixels, that are not defined in the table of class definition, are filled after classification by spectral matching using the spectral similarity value.

Part of the table of class definition is showed below.

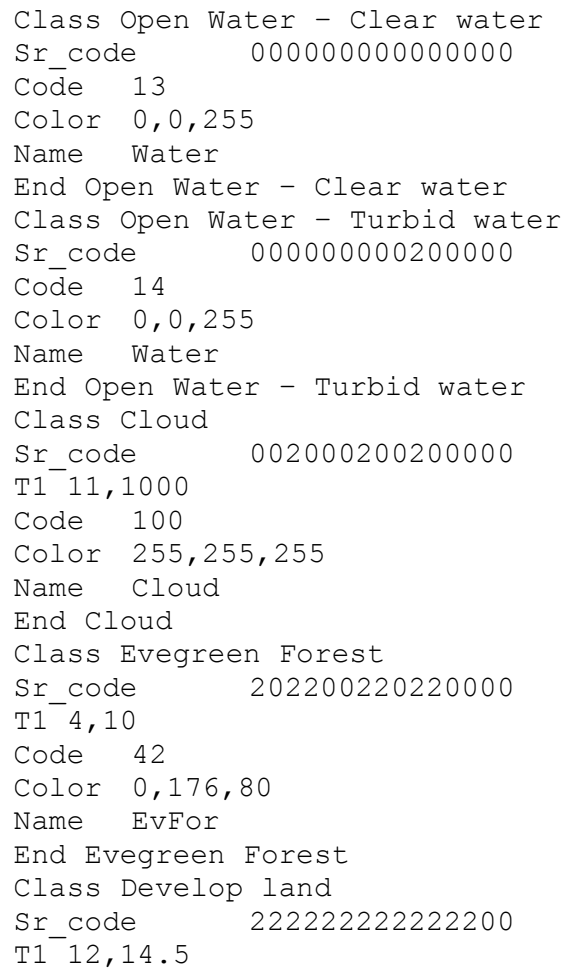

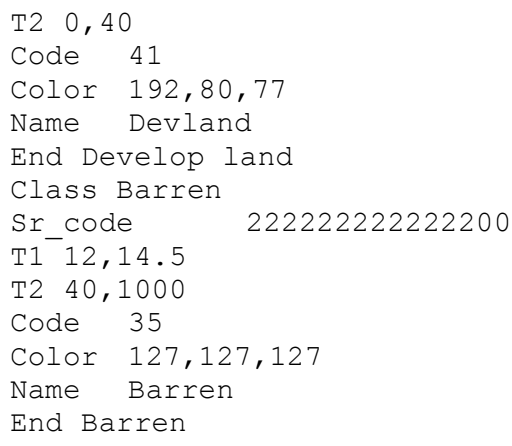

When the table of class definition is established, it can be used for classification of any Landsat 8 OLI surface reflectance scenes. Figure 3 shows result of automated classification of land cover for the scene LC81270452013352LGN00 and LC81270452015182LGN00.

\section{DISCUSSION}

The concept of automated classification proposed in this paper is based on simplified spectral patterns. The spectral pattern in general and simplified spectral pattern in particular can be considered as image invariant, which does not vary with location and time. This method of classification differs from conventional supervised classification technique, in that training is required for every scene and training data is very subjective and depends very much on skill of an operator. The automated classification by SSP requires development of class definition table. However, after the class definition table has been established, it can be applied for any other Landsat OLI scenes. Because classification of land cover is carried out using spectral pattern, so fine objects can be well classified.

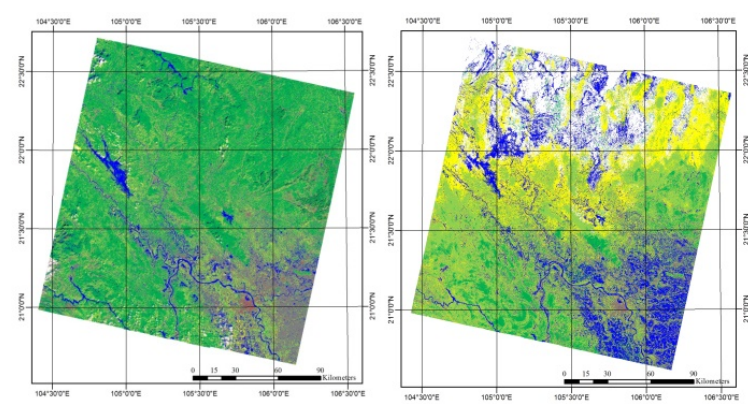

Figure 3. Classified image of the scene LC81270452013352LGN00 (left) and LC81270452015182LGN00 (right).

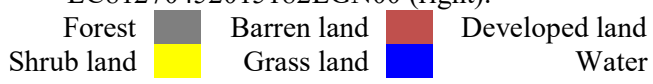

Figure 4 shows example of classification of small islands of Sword Lake of Hanoi. 


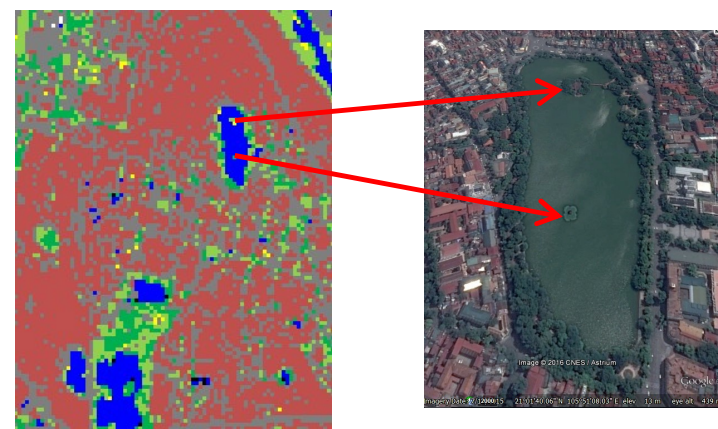

Figure 4. Good classification of small object by SSP concept. Example with Ngoc Son temple and Thap Rua of the Sword lake in Hanoi. Classified image (left) and image from the GoogleEarth (right)

Figure 5 shows very fine classification of small pathways in crop field.

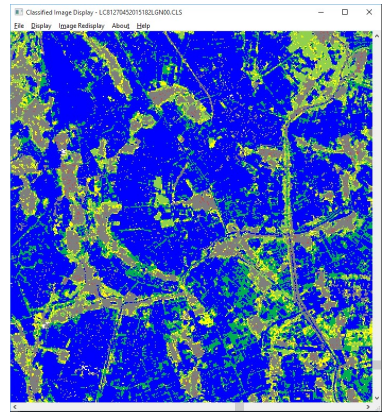

Figure 5. Very detail linear feature can be classified. Example for pathways in rice field

Theoretically, surface reflectance of a stable object does not change for a given remote sensor. However, in practice we observe slight changes of surface reflectance of built up in Hanoi when we compare data collected in 2013 and 2015. The changes of surface reflectance causes slight changes in classification results as shown in Figure 6.

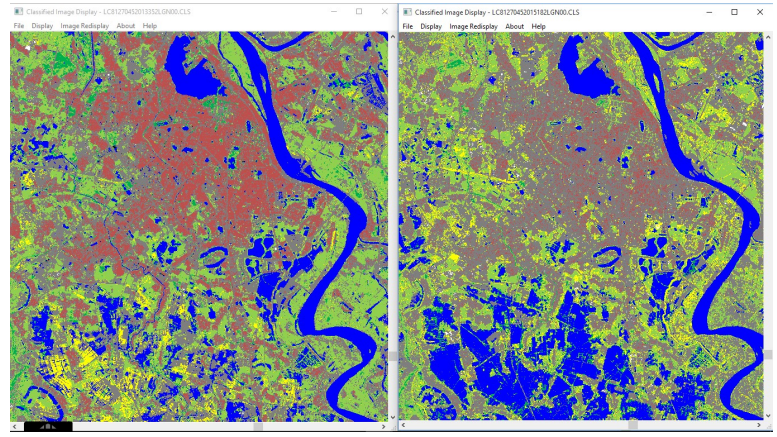

Figure 6. Comparison of automated classification results of scenes LC81270452013352LGN00 (left) and LC81270452015182LGN00 (right).

The scene LC81270452013352LGN00 was observed in Dec. 18,2013 . This is dry season for Hanoi area so rice field is after harvest and appears as barren land. The scene LC81270452015182LGN00 was observed in Jul. 1, 2015. This time is rainy season so we can see a lot of water surface in classification result. These parcels should be classified as cropland. Seasonal change of land cover causes difficulties in automated classification in that only static component of land cover is taken into consideration while dynamic component can be retrieved only if we use temporal data series. Inconsistency of surface reflectance product causes incorrectness in classification of objects, which have the same simplified spectral pattern. For example, in Figure 6, developed land of Hanoi is classified more to barren land for the scene of 2015, while for image in 2013, the developed land is correctly classified.

\section{CONCLUSION}

The paper presents the first attempt of automation of classification of land cover using the provisional surface reflectance product of Landsat 8 OLI. The study has been conducted in Hanoi city and surrounding area. The automation relies on the table of class definition in which every land cover object is define by parameters such as simplified spectral patterns, TRRI and ratios among spectral bands. The table of class definition, when has been developed, can be used for any Landsat 8 OLI surface reflectance product. The automation of classification can help mapping land cover, which reflects physical status of ground in the time of observation. To develop final land cover map there is a need to combine more single date land cover map created within one or two year period depending on availability of cloud free scenes.

\section{ACKNOWLEDGEMENTS}

This research is funded by the Vietnam National Foundation for Science and Technology Development (NAFOSTED) under grant number 105.08-2014.14.

\section{REFERENCES}

Duong N. D., 1998. Total reflected radiance index - An index to support land cover classification. Proceedings of the $18^{\text {th }}$ Asian conference on remote sensing, Manila, Philippines.

Duong, N. D. (2000). Land cover category definition by image invariants for automated classification. International archives of photogrammetry and remote sensing, 33(B7), 985-992.

Homayouni, S., \& Roux, M. (2004, July). Hyperspectral image analysis for material mapping using spectral matching. In ISPRS Congress Proceedings.

Huth, J., Kuenzer, C., Wehrmann, T., Gebhardt, S., Tuan, V. Q., \& Dech, S. (2012). Land cover and land use classification with TWOPAC: Towards automated processing for pixel-and objectbased image classification. Remote Sensing, 4(9), 2530-2553.

Syed, S., Dare, P., \& Jones, S. (2005, September). Automatic classification of land cover features with high resolution imagery and lidar data: an object-oriented approach. In Proceedings of SSC2005 spatial intelligence, innovation and praxis: the national biennial conference of the spatial sciences institute(pp. 512-522). Melbourne: Melbourne: Spatial Science Institute.

Turner, B. L., Skole, D. L., Sanderson, S., Fischer, G., Fresco, L., \& Leemans, R. (1995). Land-use and land-cover change. Science/Research Plan. Global Change Report (Sweden). 
The International Archives of the Photogrammetry, Remote Sensing and Spatial Information Sciences, Volume XLI-B8, 2016 XXIII ISPRS Congress, 12-19 July 2016, Prague, Czech Republic

USGS 2015. Landsat Surface Reflectance High Level Data Products. http://landsat.usgs.gov/cdr_lsr.php

Verhegghen, A., Defourny, P., Ernst, C., \& Beuchle, R. (2010). Automated land cover mapping and independent change detection in tropical forest using multi-temporal high resolution data set. International Archives of the Photogrammetry, Remote Sensing and Spatial Information Sciences, 38. 